\title{
Dispersion-Corrected Density Functional for the Correct Description on Regioselectivity Trends in Heck-Mizoroki Reaction Catalyzed by Anionic (N-Heterocycle Carbene)-Palladium Complexes
}

\author{
Vitor H. M. Silva, Oscar Navarro \& Ataualpa A. C. Braga
}

\section{Introduction}

Palladium-catalyzed cross-coupling reactions are an important field in organic synthesis and organometallic chemistry. Its importance was recognized in 2010 by awarding Richard Heck, Eiichi Neigishi and Arika Suzuki the Nobel Prize of Chemistry, for the prominent contribution to this field. ${ }^{1}$ The Heck-Mizoroki (HM) reaction is an excellent tool for $\mathrm{C}-\mathrm{C} \mathrm{sp}^{2}$ bond construction. ${ }^{2}$ Historically, phosphineshave been used as main ligands for palladium complexes furthering high catalytic activity in HM process, in particular cases, with excellent regio- and stereoselectivity control. ${ }^{3}$ In past decade, the N-heterocyclic carbenes (NHCs) emerged as new class of ligands in a number of crosscoupling reactions. ${ }^{4}$

The NHCs are strong $\sigma$-donors ligands, thus they are capable to provide better stability to transition metal complexes with highest oxidation state. Furthermore, NHCs are usually more sterically demanding than phosphines. The N-substituted pendants groups from central imidazole ring can play an important role in the reactivity and selectivity of catalyzed systems. Recently, density functional calculations were used to rationalize the role of the ionic (NHC)PdCl complex as pre-catalyst in a $\mathrm{HM}$ coupling reaction involving $\mathrm{PhBr}$ and styrene in the presence of $\mathrm{HCO}_{3}^{-}$as base (Scheme 1). ${ }^{5} \mathrm{In}$ this case, computational studies were applied to a model system, in which the two SIPr groups ( $\mathrm{SlPr}$ =2,6-diisopropylphenyl) were replaced by Me groups $(\mathrm{Me}=$ methyl $)$. Although this model system was able to provide new insights from the electronic influence of the NHC ligand in the reactivity and selectivity in HM coupling, the influence of the steric bulk from the real system over the selectivity of the catalyzed system still remains unclear.

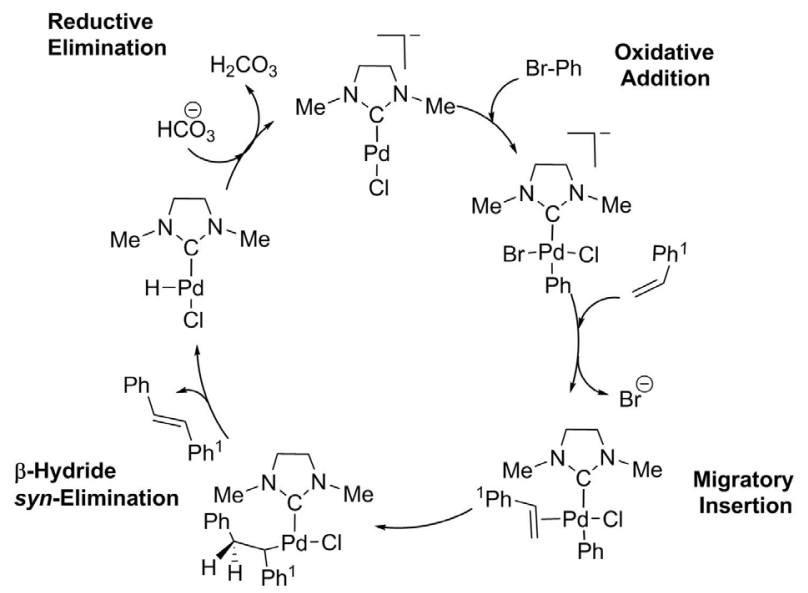

Scheme 1. Plausible HM catalytic cycle for the (NHC)PdCl- The model system was constructed using $\mathrm{Me}$ groups as $\mathrm{N}$-substituted in NHC ligand. 
Computational studies have indicated that a correct description of dispersion interactions is crucial for modeling hindered phosphine $\left(\mathrm{PPh}_{3}\right)$ ligands in Pd-catalyzed cross-coupling reactions. ${ }^{6}$ Recently, Schoenebeck and collaborators showed that dispersion forces are key controlling factor to the correct description of the oxidative addition pathways involving crowded trialkyl substituent in palladium complexes. ${ }^{7}$ Norrby and Sigman performed theoretical calculations for the enantioselective Heck-Matsuda reaction using a $N, N$ PyOx ligand with terc-butyl substituent. Only dispersioncorrected density functionals were able to provide the correct stereochemistry of the product. ${ }^{8}$

Herein, we performed a computational study based on the density functional theory (DFT) to investigate how the real (NHC)PdCl- pre-catalyst influences the HM mechanism reaction. The main goal of this work is to analyze the regioselectivity trends by different exchangecorrelation density functionals.

\section{Methods}

All electronic structure calculations were carried out within the DFT formalism using Gaussian09 suite of quantum chemical programs..$^{9-10}$ Geometries optimizations, without any symmetry restriction were carried out in gas-phase using the GGA hybrid functionals $\mathrm{PBE0}^{11}$ and $\mathrm{B} 3 \mathrm{LYP}^{12}$. The dispersion-corrected local and hybrid functional meta-GGA M06L ${ }^{13}$ and $\mathrm{M} 06^{14}$ also were used in the current study, because these approaches have been proved to be reliable methods for transition metal kinetics-thermochemistry. The long-range-corrected hybrid GGA functional $\infty \mathrm{B} 97 \mathrm{XD}^{15}$ with the Grimme's empirical damped par-wise dispersion terms (DFT-D2) was also explored. ${ }^{16}$ The SDD basis set was adopted to $\mathrm{Pd}^{17}$ and the 6-31G(d) basis set for the remaining atoms. This basis set approach is denoted as BS1 $(6-31 \mathrm{G}(\mathrm{d})$, SDD (Pd)). Frequency calculations were performed in order to verify the nature of all stationary points on the potential energy surface (PES). The vibrational analysis was performed within the harmonic approximation with thermochemical data calculated at $298 \mathrm{~K}$ and 1 atm. Intrinsic reaction coordinate (IRC) $)^{18}$ calculations were used to connect reactants to products. Solvents effects were take into account with the continuum solvation model $\mathrm{SMD}^{19}$ with DMF as the solvent. The
SMD calculations were performed as single-point energies calculations (SMD) on the optimized gas-phase geometries. In specific cases, single-point energies have been calculated using the SMD-6-311+G(d), SDD(Pd) level of theory. This basis set approaches denoted as BS2 (6-311+G(d), SDD (Pd)). All energies are presented in $\mathrm{kcal} / \mathrm{mol}$ with respect to the lowest energy structure, unless otherwise specified.

\section{Results and Discussion}

The regioselective proposal for the catalyzed HM arylation is depicted in Scheme 2. The real (NHC)PdClcomplex is the catalyst. The starting aryl-palladium (II) complexes has two possible configurations, 1 and 2, with the $\mathrm{Ph}$ group $(\mathrm{Ph}=$ aryl $)$ trans or cis to the $\mathrm{NHC}$ ligand, respectively. The stereoisomer 1 was found to be $10 \mathrm{kcal} /$ mol higher than 2 at free energy surface, at SMD-PBE0/ BS1 level of theory. It is noted the strongest NHC trans influence over the aryl group, increasing the energy of the intermediate 1. Besides the thermodynamic driving force in the isomerization of 1 towards to 2 , we were unable to find the transition state for the isomerization via an associative or a dissociative process. A Berry pseudorotation mechanism was discarded, once a hindered steric bulk of the SIPr groups in imidazole ring is present.

In addition, it is well-know that a cis-trans isomerization is accelerated, ${ }^{1,6 b, 8,21}$ when chlorine and basic ligands are present in HM catalyzed systems. Therefore, it is expected that the isomerization process would be faster than the migratory insertion of olefin into the starting intermediates 1 and 2. Consequently, the selectivity-determining step is the migratory insertion under Curtin-Hammet condition, in which the regioselectivity is determined solely by the relative energies of the competing insertion migratory transition states..$^{20}$ The transition states TS1 and TS3 are associated to the linear product, while the TS2 and TS4 yield the branched product. It is worthy to mention that only the linear product is detected by the experimental work, see the catalytic cycle in Scheme 1. The search for linear and branched transition states, having the full ligand SIPr, was made using DFT calculations. The dispersionfree density functional, PBE0 and B3LYP, and density functionals that take in account the dispersion forces (M06L, M06 and $₫$ B97XD) were used. 


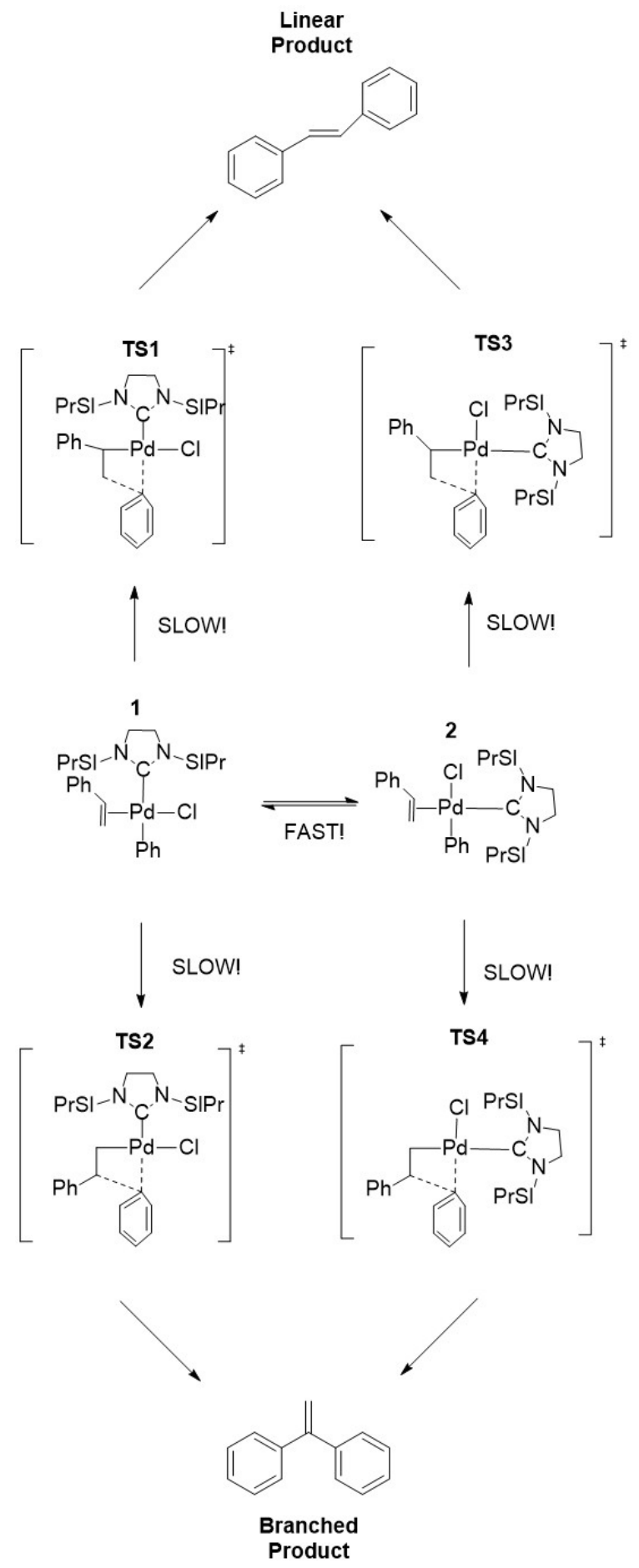

Scheme 2. $\beta$ - and $y$ - insertion migratory pathways starting through an isomerization between aryl-palladium (II) alkenes intermediates

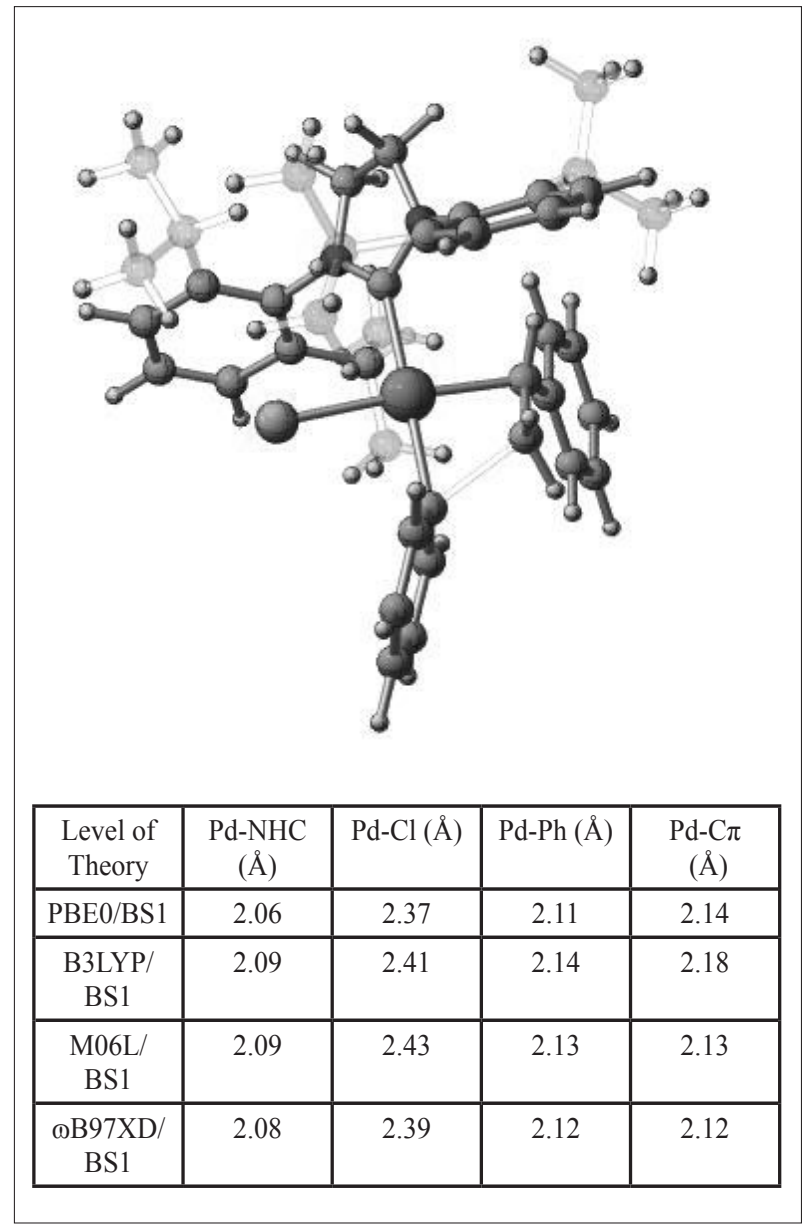

Figure 1.Crucial optimized features of TS1.

The optimized TS1 associated to linear products is illustrated in Figure 1. Its molecular structure clearly shows the C-C bond formation via four-membered arrangement. The dispersion has no significant impact on TS arrangement with respect to the calculations obtained using the model system. The Pd-NHC and Pd$\mathrm{C} \pi$ bonds in NHC model $(\mathrm{R}=\mathrm{Me})$ were found to be 2.04 and 2.10 $\AA$, respectively, at M06L/6-31+G(d), SDD (Pd) level of theory. For the real system, this bond distance is about 2.09 and $2.10 \AA$, respectively, at M06L/BS1. The geometries obtained using M06L and wB97XD dispersion-corrected functionals are quite close; except in the case of $\mathrm{Pd}-\mathrm{Cl}$ distance where the difference was $0.4 \AA$ shorter using wB97XD functional. Calculations 
based on PBE0 and B3LYP showed larger differences compared to the dispersion-corrected methods.

The electronic influence of $\mathrm{NHC}$ on the reaction regioselectivity was explored with Me groups in the NHC ligand, ${ }^{5}$ In the model system, the $\beta$-carbon addition was preferential, and the final product was in agreement with the experimental reports. The regioselectivity trends can be understood in terms of how metal-ligand bond changes during the course of the $\mathrm{C}-\mathrm{C}$ bond formation. ${ }^{20}$ As the new bond is forming, relative to the $\beta$-carbon $(\mathrm{C} \pi)$, a negative charge is generated in the neighbor carbon, which results in a stronger interaction with the palladium center. In the linear transition states, this carbanion is stabilized by conjugation with the styrene, decreasing its trans influence. On the other hand, in the branched transition state structures, the negative charge on alkyl group is free causing strongest trans influence over the ligands. The NHC ligand, a stronger $\sigma$-donor ligand and $\pi$-acceptor, once the trans to the localized carbanion, it is expect a destabilization more pronounced in the transition states structures than to exerted by chlorine moiety, ligand with weak trans influence. Therefore, it is expected that TS3 and TS4 have higher energy than the TS1 and TS2. In addition, the charge separation stabilized by conjugation into TS1 and TS3 transition states structures explains the electronic influence on regioselectivity trends to the linear product. However, into the real NHC catalyst, the dispersion forces are correlated with the electronic influence of ligand, thus both chemical properties contributed to regioselectivity of the reaction.

The computed enthalpies and Gibbs free energies in DFM solution related to the insertion transition states are shown in Table 1. The PBE0 and B3LYP density functional favor the branched product via the transition state TS2, in total disagreement with the regioselectivity reported in the experimental work. ${ }^{5}$ The M06L method improves the energies, decreasing in 3 and $5 \mathrm{kcal} / \mathrm{mol}$, the stability of TS2 as predicted by the PBE0 and the B3LYP functionals, however, the free energies barriers stills report the branched configuration as preferential product. Only the long-range-corrected B97XD method favors the linear transition state TS1, in agreement with the experimental data. M06 singlepoints calculations at the B3LYP optimized geometries are a common tool in computational studies involving metal transition catalyzed systems. ${ }^{14}$ In the preset study, the B3LYP/BS1/M06/BS2 approach failed in predicting the right regioselectivity. In contrast, the $\omega \mathrm{B} 97 \mathrm{XD} /$ BS1/M06/BS2 approach was able to properly describe the experimental regioselectivity. These results show the importance of using computational approaches that account for dispersion effects. Such approaches should be use in geometry optimizations, mainly for crowded organometallics catalysts. Importantly, no matter whether dispersion is included or not, the highest energies are obtained for TS3 and TS4 transition sates, showing that the strong electronic influence of NHC ligand can overcome the attractive intramolecular dispersion forces

Table 1.Relative enthalpies and free energies in DFM solution. The energies are computed with respect to the lowest energy transition state structure.

\begin{tabular}{|c|c|c|c|c|c|c|c|c|}
\hline \multirow[t]{2}{*}{ Level of Theory } & \multicolumn{4}{|c|}{$\Delta \mathrm{H}_{\mathrm{DMF}}(\mathrm{kcal} / \mathrm{mol})$} & \multicolumn{4}{|c|}{$\Delta \mathrm{G}_{\mathrm{DMF}}(\mathrm{kcal} / \mathrm{mol})$} \\
\hline & TS1 & TS2 & TS3 & TS4 & TS1 & TS2 & TS3 & TS4 \\
\hline PBE0/BS1 & 3.0 & 0.0 & 7.8 & 10.2 & 4.1 & 0.0 & 8.7 & 10.9 \\
\hline B3LYP/BS1 & 4.2 & 0.0 & 8.6 & 10.9 & 6.0 & 0.0 & 10.2 & 12.0 \\
\hline M06L/BS1 & 0.0 & 0.4 & 4.4 & 7.8 & 1.1 & 0.0 & 6.5 & 10.9 \\
\hline ๓B97XD/BS1 & 0.0 & 0.6 & 5.5 & 6.7 & 0.0 & 0.8 & 7.2 & 7.7 \\
\hline $\begin{array}{c}\oplus \mathrm{B} 97 \mathrm{XD} / \mathrm{BS} 1 / / \\
\oplus \mathrm{B} 97 \mathrm{XD} / \mathrm{BS} 2\end{array}$ & 0.0 & 0.8 & 5.8 & 7.6 & 0.0 & 1.0 & 7.5 & 8.5 \\
\hline $\begin{array}{c}\text { B3LYP /BS1// } \\
\text { M06/BS2 }\end{array}$ & 0.7 & 0.0 & 3.6 & 6.5 & 2.5 & 0.0 & 5.2 & 7.6 \\
\hline $\begin{array}{c}\oplus \mathrm{B} 97 \mathrm{XD} / \mathrm{BS} 1 / / \\
\mathrm{M} 06 / \mathrm{BS} 2\end{array}$ & 0.0 & 0.2 & 3.9 & 6.7 & 0.0 & 0.5 & 5.7 & 7.6 \\
\hline
\end{tabular}


of real ligands. Furthermore, our results suggest that the regioselectivity in the present system follows a typical Harper mechanism ${ }^{21}$, wherein the major product, in this case the linear configuration, arises from a preceding minor intermediate, the alkene-palladium (II) complex 1.

\section{Conclusions}

In conclusion, density functionals that account for dispersion correction are fundamental to predict the correct regioselectivity in insertion migratory step of the NHC-catalyzed Heck-Mizoroki reaction discussed. Herein, we explored the influence of real NHC ligand bulk, an extension of computational studies on model NHC ligand with methyl ligands reported in the literature. The use of B3LYP and PBE0 functionals result in a strikingly different regioselectivity compared to the experimental prediction. Only hybrid dispersioncorrected $₫ \mathrm{~B} 97 \mathrm{XD}$ and meta-GGA M06 functionals were capable to describe the influence of real ligand to the reaction regioselectivity. We expected with complete elucidation on the selectivity-controlling this catalyzed system, to start investigations based on theoretical design of new NHC ligands.

\section{Acknowledgments}

The V.H.M.S. is thankful for scholarship grant \#2013/04813-6, São Paulo Research Foundation, and appreciates the generous help for Ana P. de Lima Batista with manuscript editing and helpful suggestions. The authors are grateful for the financial support given from the grant \#2015/01491-3, São Paulo Research Foundation.

\section{References}

1. C. C. C. Johansson Sechurn, M. O. Kitching, T. J. Colacot, V. Snieckus, Angew.Chem. Int. Ed., 51, 5062 (2012).

2. F. R. Heck, J. P. Nolley, J. Org. Chem. 37, 2320 (1972) b)T. Mizoroki, K. Mori, A. Ozaki, Bull. Chem. Soc. Jpn., 44, 581 (1971).

3. M. Garcia-Melchor, A. A. C. Braga, Llédos A., G. Ujaque, Acc. Chem. Res., 46, 2626 (2013).

4. G. C. Fortman, S. P. Nolan, Chem. Soc. Rev., 40, 5151 (2010).

5. D. Guest, V. H. Menezes da Silva, A. P. De Lima Batista, M. Roe, A. A. C. Braga, O. Navarro, Organometallics, 34, 2463 (2015).

6. a) S. Grimme, P. R. Schreiner, Angew.Chem. Int. Ed., 50, 11794 (2011).b) M. S. G. Ahlquist, P. -O., Norrby, Angew.Chem. Int. Ed., 50, 12639 (2011).

7. E. Lyngvi, I. A. Sanhueza, F. Schoenebeck, Organometallics, 34, 805 (2015).

8. L. Xu, M. J. Hilton, X. Zhang, P. -O. Norrby, Y. -D. Wu, M. S. Sigman, O. Wiest, J. Am. Chem. Soc., 136, 1960 (2013).

9. a) P. Hohenberg, W. Kohn Phys. Rev. 136, B864, (1964). b) W. Kohn, L. Sham Phys. Rev. 140, A1113, (1965).

10. M. J. Frisch et al.Gaussian09, Revision D.01, Gaussian Inc., Wallingford, CT (2009).

11. J. P. Perdew, K. Burke, M. Ernzerhof, Phys. Rev. Lett., 77, 3865 (1996).

12. A. D. Becke,, J. Chem. Phys. 98, 5648 (1993). b) W. Kohn, A. D. Becke, R. G. Park, J. Phys. Chem. 100, 12974, (1996). c) C. Lee, W. Yang, R. G. Parr, Phys. Rev. B, 37, 785, (1988).

13. Y. Zhao, D. G. Truhlar, J. Chem. Phys., 125, 194101 (2006).

14. Y. Zhao, D. G. Truhlar, Theor. Chem. Acc. 120, 215 (2008) b) Y. Zhao, D. G. Truhlar, Acc. Chem. Res., 41, 157 (2008).

15. J. -D. Chai, M. Head-Gordon, Phys. Chem. Chem. Phys. 10, 6615 (2008).

16. Grimme, J. Comp. Chem. 27, 1787 (2006).

17. D. Andrae, U. Huermann, M. Dolg, H. Stoll, H. Preu, Theor. Chim.Acta, 77, 123, 1991.

18. H. P. Hratchian, H. B. Schlegel, J. Chem. Theory Comput., 1, 61 (2005).

19. A. V. Marenich, C. J. Cramer, D. G. Truhlar, J. Phys. Chem. B, 113, 6378 (2009).

20. C. Bäcktorp, P. -O.Norrby, J. Mol. Catal. A: Chem., 328, 108 (2010).

21. S. T. Henriksen, P. -O. Norrby, P. Kaukoranta, P. G. Andersson J. Am. Chem. Soc., 130, 10414 (2008).

Vitor H. Menezes da Silva ${ }^{a^{*}}$, Oscar Navarro ${ }^{b}$ \& Ataualpa A. C. Braga ${ }^{\circ}$

anstituto de Química, Departamento de Química Fundamental, Universidade de São Paulo, SP, Brasil.

${ }^{b}$ Department of Chemistry, University of Sussex, Brighton, BN1 9QJ, United Kingdom

*E-mail: vhugomenezes@iq.usp.br 